\title{
Comparison of 5-year outcomes between trabeculectomy combined with phacoemulsification and trabeculectomy followed by phacoemulsification: a retrospective cohort study
}

\author{
Shogo Arimura, Kentaro Iwasaki, Yusuke Orii, Yoshihiro Takamura and Masaru Inatani
}

\begin{abstract}
Background: We aimed to compare the outcomes of trabeculectomy combined with phacoemulsification and those of trabeculectomy followed by phacoemulsification.

Methods: A total of 141 patients with primary open-angle glaucoma, exfoliation glaucoma, and glaucoma secondary to uveitis glaucoma who underwent trabeculectomy followed by $(n=48)$ or combined with $(n=93)$ phacoemulsification were included. We analyzed data collected from the Collaborative Bleb-Related Infection Incidence and Treatment Study, a prospective cohort study conducted in 34 clinical centers that included 1249 eyes. The main outcome was the cumulative probability of success based on intraocular pressure (IOP) within 5 years. Surgical failure was defined as a case in which additional glaucoma surgery is required or one of the following criteria are met: preoperative IOP $>21(\mathrm{~A}),>18$ (B), or $>15 \mathrm{mmHg}(\mathrm{C})$. The secondary outcomes were cumulative probability of success, risk factors of surgical failure, and $\Delta$ visual acuity. However, the data on phacoemulsification during the 5-year follow-up were censored.
\end{abstract}

Results: No significant difference was found in the cumulative probability of success as the main outcome. When the data on phacoemulsification during the 5-year follow-up were censored, the probabilities of success of trabeculectomy followed by phacoemulsification were significantly higher for criteria $\mathrm{A}(p=0.02), \mathrm{B}(p<0.01)$, and $C(p<0.01)$. Lower preoperative IOP, younger age, and trabeculectomy combined with phacoemulsification were associated with poorer outcome. Trabeculectomy followed by phacoemulsification had significantly worse $\Delta$ logMAR visual acuity at 6 and 12 months $(p<0.01)$.

Conclusion: The cumulative probability of success after trabeculectomy combined with or followed by phacoemulsification remained unchanged. Combining phacoemulsification with trabeculectomy adversely affected the cumulative probability of success after trabeculectomy. The visual acuity improvements observed in the early postoperative period after combining phacoemulsification with trabeculectomy disappeared within 5 years.

Keywords: Trabeculectomy, Phacoemulsification, Long-term outcome

\footnotetext{
*Correspondence: inatani@u-fukui.ac.jp

Department of Ophthalmology, Faculty of Medical Science, University of

Fukui, Yoshida, 23-3 Simoaizuki, Matsuoka, Fukui, Japan
} 


\section{Background}

Trabeculectomy has been the standard filtering surgery for patients with glaucoma due to uncontrollable intraocular pressure (IOP) [1]. When trabeculectomy is performed in patients with glaucoma, the best timing to perform cataract surgery is difficult to determine because the cataract frequently progresses after trabeculectomy [2-4] and because phacoemulsification is known to adversely affect IOP control after trabeculectomy [5-7]. Recently, we also reported that phacoemulsification performed in patients with a history of trabeculectomy led to poorer surgical outcomes of trabeculectomy alone [8], and trabeculectomy combined with phacoemulsification has poorer surgical outcomes than trabeculectomy alone $[9,10]$. In addition, the numbers of patients with glaucoma and cataract are increasing with the aging of society and population growth worldwide [11]. This situation has caused increasing concern about the best timing to perform cataract surgery for patients with a history of trabeculectomy. However, no studies conducted in large cohorts and over long periods have assessed surgical outcomes between trabeculectomy followed by phacoemulsification and trabeculectomy combined with trabeculectomy.

In the present study, we analyzed data collected from the Collaborative Bleb-Related Infection Incidence and Treatment Study (CBIITS), a prospective cohort study conducted in 34 clinical centers that included 1249 eyes [12]. The CBIITS investigated the incidence, severity, and prognosis of bleb-related infections after trabeculectomy with mitomycin $\mathrm{C}$ treatment for 5 years. Therefore, the present study aimed to compare long-term outcomes between trabeculectomy followed by phacoemulsification and trabeculectomy combined with phacoemulsification and to identify the risk factors of surgical failure. By clarifying the outcomes, we aimed to help determine whether cataract surgery should be performed at the same time as trabeculectomy or later in clinical settings.

\section{Methods}

\section{Patient selection}

The CBIITS is a multicenter prospective cohort study that aimed to investigate the incidence and risk factors of bleb-related infection. The investigators from the Japan Glaucoma Society voluntarily participated in the CBIITS, in which 34 clinical centers were enrolled as participants. Institutional review board approval from each clinical center was obtained, and written informed consent was obtained from all the participants. The protocol conformed to the tenets of the Declaration of Helsinki. The postoperative ophthalmologic examinations were conducted every 6 months up to 5 years. The CBIITS enrolled 1249 eyes of 1249 patients for 2 years, and the enrollment was completed on March 31, 2007. The study finally included 1098 eyes treated with trabeculectomy.

The institutional review board of Fukui University Hospital, Fukui, Japan, approved the present study, and 141 eyes of 141 patients with primary open-angle glaucoma, exfoliation glaucoma, and glaucoma secondary to uveitis who had participated in the CBIITS were included. We notified and disclosed research information on the homepage to guarantee the opportunity for research participants to refuse. The enrolled patients were divided into two groups, the "trabeculectomy followed by phacoemulsification" group and the "trabeculectomy combined with phacoemulsification" group. "Trabeculectomy followed by phacoemulsification" referred to the performance of lens extraction with phacoemulsification during the 5-year follow-up period after trabeculectomy. In the CBIITS, the local investigators at each center decided the indications for surgery, selection of operative procedure, operative technique, and application of postoperative medication or additional glaucoma treatment, either medically or surgically. However, trabeculectomy was usually performed with $0.04 \%$ mitomycin and a fornix- or limbus-based conjunctival incision. Phacoemulsification was performed with a clear corneal or superior conjunctival incision.

In the present study, we included Japanese patients with primary open-angle glaucoma, exfoliation glaucoma, and glaucoma secondary to uveitis (with the anterior chamber angle assessed using slit-lamp biomicroscopy and gonioscopy with an angle mirror, and the fundus evaluated using ophthalmoscopy or fundus photography); who were at least 20 years of age; who underwent trabeculectomy with $0.04 \%$ mitomycin C; who had phakic eyes; and who completed the 5-year follow-up without dropouts. We excluded eyes with histories of glaucoma and vitreoretinal surgeries, rupture during phacoemulsification, and any postoperative intraocular surgeries except for glaucoma reoperation or phacoemulsification.

\section{Main outcome measure}

The primary outcome measure was the cumulative probability of success in the Kaplan-Meier survival curve analysis based on IOP. IOP was measured with Goldmann applanation tonometry every 6 months at each center during the 5-year follow-up in the CBIITS. In the present study, surgical failure was defined, before performing the data analysis, on the basis of the following IOP levels with or without antiglaucoma medications: < $20 \%$ reduction in preoperative IOP or IOP $>21 \mathrm{mmHg}$ (criterion A), IOP > $18 \mathrm{mmHg}$ (criterion B), or IOP > 15 $\mathrm{mmHg}$ (criterion C) [13]. In addition, surgical failure was declared in cases that required reoperation for 
glaucoma or had loss of light perception or low IOP $(\leq 5$ $\mathrm{mmHg}$ ). Reoperation for glaucoma was comprised of bleb needling $>6$ months after trabeculectomy, bleb revision, or additional glaucoma surgery. Laser suture lysis or bleb needling within 6 months after trabeculectomy was not considered as surgical failure because it was a part of the postoperative management for trabeculectomy.

\section{Secondary outcome measures}

The secondary outcomes included three factors. The first factor was the cumulative probability of success in the Kaplan-Meier survival curve analysis, with the same criteria as those of the primary outcome, but the data on phacoemulsification during the 5-year follow-up were censored. The second factor was the risk factors of failure in the first secondary outcome. The third factor was $\Delta$ visual acuity (the postoperative visual acuity minus the preoperative visual acuity) during the 5-year follow-up. Visual acuity aggregated in the form of decimal visual acuity with the Landolt chart was converted to a logarithm of the minimum angle of resolution (logMAR) visual acuity in the statistical analysis.

\section{Sample size}

In a previous study [11], the cumulative probability of success after trabeculectomy followed by phacoemulsification appeared to be superior to that of trabeculectomy combined with phacoemulsification when the data on phacoemulsification during the 5-year follow-up were censored. Therefore, we assumed proportional hazards and designed the sample size by using the method of Lakatos et al. [14] to test the difference between the survival functions of the two groups. If the difference in surgical failure rate between the two groups was $\geq 25 \%$ with a two-sided significance level of 0.05 and a power of 0.8 , the estimated sample size of at least 105 eyes was considered as essential to detect a significant difference between the two groups.

\section{Statistical analyses}

SPSS version 26.0 (IBM Institute, Inc. Chicago, IL, USA) was used for the statistical analysis. Univariate analyses were performed using the Wilcoxon non-parametrical and chi-square tests for baseline characteristics. The Wilcoxon non-parametrical test with Bonferroni correction was used to determine the $\Delta$ visual acuity between the two groups. The 5-year cumulative probability of surgical failure was analyzed using the Kaplan-Meier survival curves and log-rank test. The Cox proportional hazards regression model was used to determine the risk factors of surgical failure. $P$ values were considered statistically significant if less than 0.05 .

\section{Results}

Baseline characteristics

Table 1 shows the baseline characteristics. A total of 48 eyes were treated with trabeculectomy followed by phacoemulsification, whereas 93 eyes were treated with trabeculectomy combined with phacoemulsification. Significant differences were observed in the number of eyes with secondary glaucoma due to uveitis $(p=0.02)$, preoperative $\log$ MAR visual acuity $(p<0.01)$, and the base of the conjunctival flap $(p=0.03)$ between the two groups. Preoperative IOP (mean $\pm \mathrm{SD}$ ) was not significantly different between the "trabeculectomy followed by phacoemulsification" group $(24.0 \pm 10.1 \mathrm{mmHg})$ and "trabeculectomy combined with phacoemulsification" group $(24.4 \pm 8.0 \mathrm{mmHg} ; p=0.35)$.

\section{Primary outcome}

The results of the Kaplan-Meier survival curve analysis between the "trabeculectomy followed by phacoemulsification" and "trabeculectomy combined with phacoemulsification" groups for criteria A, B, and C are shown in Fig. 1. No significant differences were observed between the two groups in any criteria. For criterion $\mathrm{A}$, the cumulative probabilities of success of trabeculectomy followed by phacoemulsification and trabeculectomy combined with phacoemulsification at 5 years were 52.1 and $43.0 \%(p=0.22)$, respectively. For criterion B, the 5year cumulative probabilities of success of trabeculectomy followed by phacoemulsification and trabeculectomy combined with phacoemulsification were 39.6 and $36.6 \%(p=0.44)$, respectively. For criterion $C$, the 5 -year cumulative probabilities of success of trabeculectomy followed by phacoemulsification and trabeculectomy combined with phacoemulsification were 29.2 and $22.6 \%$ $(p=0.14)$, respectively. Failure due to insufficient IOP reduction was observed in 18 eyes (38\%) based on criterion A, 25 eyes (52\%) based on criterion B, and 27 eyas (56\%) based on criterion $\mathrm{C}$ in the "trabeculectomy followed by phacoemulsification" group, and insufficient IOP reduction was observed in 43 eyes $(46 \%)$ based on criterion A, 49 eyes (53\%) based on criterion B, and 64 eyes $(69 \%)$ based on criterion $C$ in the trabeculectomy alone group (Table 2).

\section{Secondary outcomes}

Figure 2 shows that the "trabeculectomy combined with phacoemulsification" group had significantly lower cumulative probabilities of success for criteria A, B, and C ( $p=0.02, p<0.01$, and $p<0.01$, respectively) than the "trabeculectomy followed by phacoemulsification" group when the data on phacoemulsification during the 5-year follow-up were censored. For criterion A, the 5-year cumulative probabilities of success of trabeculectomy followed by phacoemulsification and trabeculectomy 
Table 1 Baseline characteristics

\begin{tabular}{llll}
\hline & TLE followed by Phaco & TLE combined with Phaco & $\boldsymbol{p}$ Value \\
\hline $\mathrm{n}$ & 48 & 93 & 0.197 \\
Age (years) & $64.5 \pm 12.0$ & $67.7 \pm 9.95$ & 0.86 \\
Sex, male (\%) & 56.2 & 53.8 & 0.29 \\
POAG, $\mathrm{n}(\%)$ & $31(65)$ & $50(54)$ & $7(8)$ \\
EXG, $\mathrm{n}(\%)$ & $8(17)$ & $36(39)$ & 0.17 \\
SG, $\mathrm{n}(\%)$ & $9(19)$ & $0.48 \pm 0.50$ & $0.02^{\dagger}$ \\
LogMAR VA & $0.14 \pm 0.28$ & 54.8 & $0.01^{*}$ \\
Base, fornix (\%) & 34.7 & $24.4 \pm 8.0$ & $0.03^{\dagger}$ \\
IOP (mmHg) & $24.0 \pm 10.1$ & $2.7 \pm 1.0$ & 0.35 \\
Glaucoma medication (n) & $2.3 \pm 1.1$ & 0.14 \\
\hline
\end{tabular}

TLE Trabeculectomy, Phaco Phacoemulsification, POAG Primary open-angle glaucoma, EXG Exfoliation glaucoma, SG Secondary glaucoma, Posner PosnerSchlossman syndrome, LogMAR Logarithm of minimum angle of resolution, VA Visual acuity, IOP Intraocular pressure.

${ }^{+}$Chi-square test

*Wilcoxon non-parametrical test

combined with phacoemulsification were 66.7 and $43.0 \%$ $(p=0.02)$, respectively. For criterion $\mathrm{B}$, the 5 -year cumulative probabilities of success of trabeculectomy followed by phacoemulsification and trabeculectomy combined with phacoemulsification were 64.6 and $37.6 \%(p<0.01)$, respectively. For criterion $\mathrm{C}$, the 5 -year cumulative probabilities of success of trabeculectomy followed by phacoemulsification and trabeculectomy combined with phacoemulsification were 54.2 and $22.6 \%(p<0.01)$, respectively.

\section{Risk factors of surgical failure}

The results of the Cox proportional hazards model when data on phacoemulsification during the 5-year follow-up were censored are listed in Table 3. Trabeculectomy combined with phacoemulsification was consistently associated with poorer surgical outcomes for criteria A $(p=0.01), \mathrm{B} \quad(p=0.01)$, and $\mathrm{C}(p<0.01)$. Preoperative lower IOP was associated with poorer surgical outcomes for criteria A $(p<0.01)$ and $\mathrm{B}(p=0.04)$. Younger age was associated with poorer surgical outcomes for criteria A $(p=0.02)$ and B $(p=0.04)$.

\section{$\Delta$ visual acuity}

The 5-year $\Delta$ visual acuity of the "trabeculectomy followed by phacoemulsification" and "trabeculectomy combined with phacoemulsification" groups are shown in Fig. 3. The $\Delta$ visual acuity in the "trabeculectomy followed by phacoemulsification" and "trabeculectomy combined with phacoemulsification" groups were $0.13 \pm$ 0.25 and $-0.16 \pm 0.47$ at 6 months $(p<0.01), 0.20 \pm 0.46$ and $-0.15 \pm 0.53$ at 12 months $(p<0.01), 0.06 \pm 0.29$ and
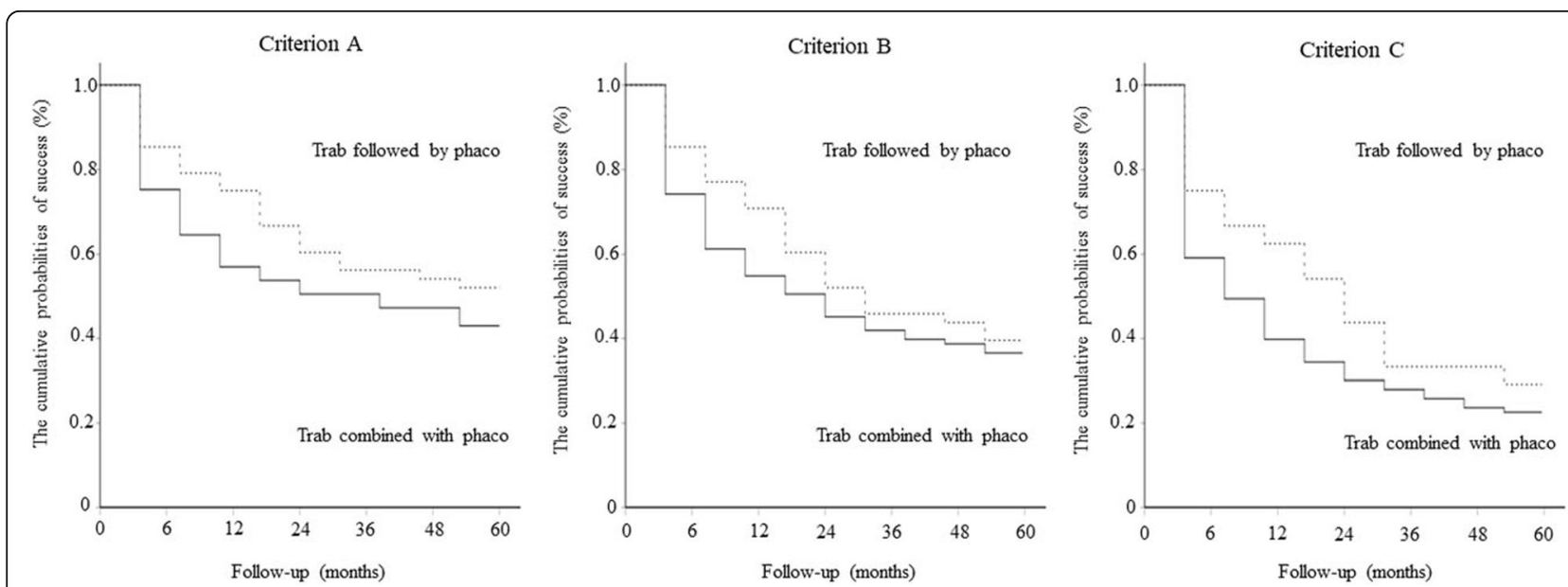

Fig. 1 Comparison of the Kaplan-Meier survival curve analysis results between the "trabeculectomy followed by the phacoemulsification" and "trabeculectomy combined with phacoemulsification" groups for criteria $\mathbf{a}$, $\mathbf{b}$, and $\mathbf{c}$. No significant differences were found between the two groups in all the criteria. The data were compared using a log-rank test. Trab = trabeculectomy; Phaco = phacoemulsification 
Table 2 Insufficient IOP reduction for surgical failure

\begin{tabular}{|c|c|c|c|}
\hline Criterion & TLE followed by Phaco & TLE combined with Phaco & $p$ Value \\
\hline & $n=48$ & $n=93$ & \\
\hline A & $n=18(38 \%)$ & $n=43(46 \%)$ & 0.32 \\
\hline B & $n=25(52 \%)$ & $n=49(53 \%)$ & 0.95 \\
\hline C & $n=27(56 \%)$ & $n=64(69 \%)$ & 0.14 \\
\hline
\end{tabular}

The data were analyzed with a chi-square test.

TLE Trabeculectomy, Phaco Phacoemulsification, IOP Intraocular pressure

$-0.03 \pm 0.60$ at 36 months $(p=0.12)$, and $0.11 \pm 0.41$ and $0.07 \pm 0.76$ at 60 months $(p=0.40)$, respectively.

\section{Discussion}

In the present study, we found no significant differences in the cumulative probabilities of success between the "trabeculectomy followed by phacoemulsification" and "trabeculectomy combined with phacoemulsification" groups. By contrast, when the data on phacoemulsification performed during the 5-year follow-up were censored, significant differences were observed for criteria $\mathrm{A}, \mathrm{B}$, and $\mathrm{C}$ between the two groups. The potential risk factors in the Cox proportional hazards model revealed that trabeculectomy combined with phacoemulsification is an independent contributor to surgical failure (criterion $\mathrm{A}: \mathrm{RR}=2.31, p=0.01$; criterion $\mathrm{B}, \mathrm{RR}=2.26, p=$ 0.01 ; and criterion $C, R R=2.23, p<0.01$; Table 3 ).

No prospective studies have been conducted in a large cohort with a long follow-up period to investigate the outcome of trabeculectomy combined with phacoemulsification. Ogata et al. prospectively reported that trabeculectomy combined with phacoemulsification $(n=25)$ resulted in inadequate IOP reduction as compared with trabeculectomy alone $(n=25)$ at 1 year [10]. Sacchi et al. retrospectively demonstrated that trabeculectomy alone $(n=40)$ achieved a higher success rate and lower mean IOP at follow-up than trabeculectomy combined with phacoemulsification $(n=27)$ for the patients with a mean follow-up period of $25.7 \pm 14.4$ months [15]. In another retrospective study, trabeculectomy combined with phacoemulsification had a lower cumulative probability of success than trabeculectomy alone for 4 years among older patients with open-angle glaucoma [11]. In a retrospective study, Donoso et al. showed that the surgical survival curve of trabeculectomy combined with phacoemulsification with intraoperative administration of 5fluorouracil had no significant difference with that of trabeculectomy followed by phacoemulsification with intraoperative administration of 5-fluorouracil [16]. The results of the present study were consistent with those of previous studies that showed that trabeculectomy combined with phacoemulsification had no significant difference in cumulative probability of success based on IOP as compared with trabeculectomy followed by
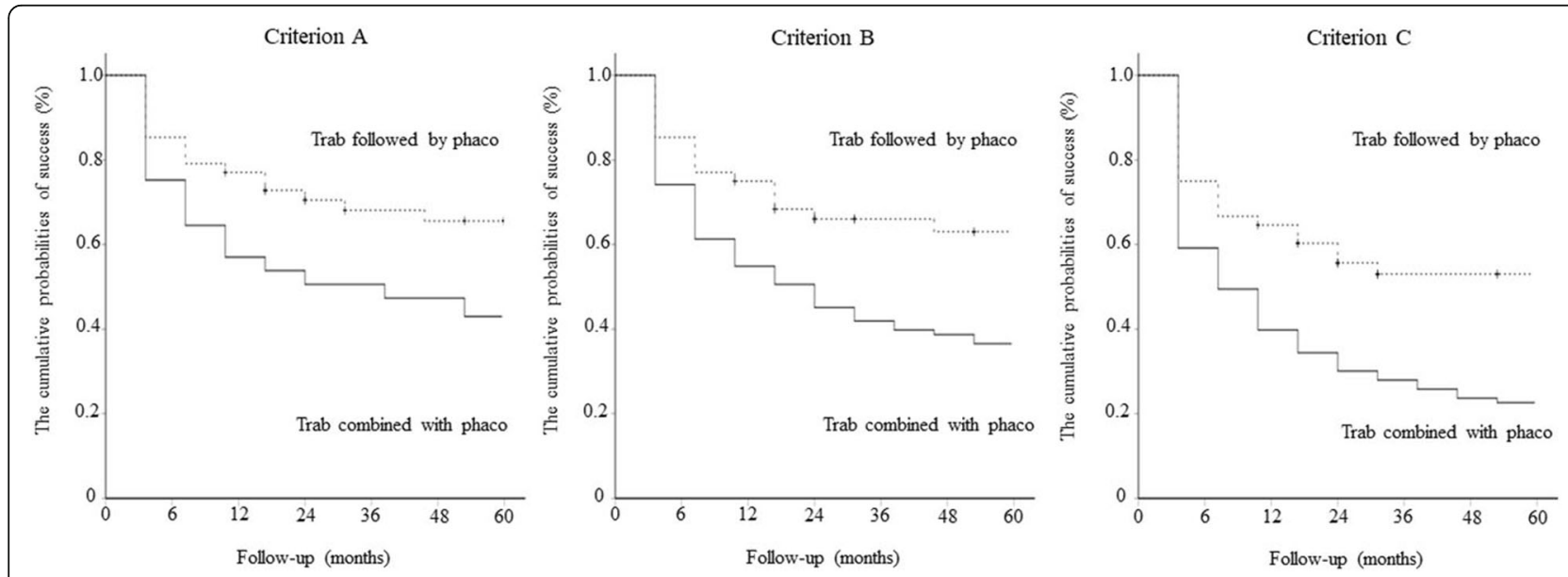

Fig. 2 Comparison of the Kaplan-Meier survival curve analysis results between the "trabeculectomy followed by phacoemulsification" and "trabeculectomy combined with phacoemulsification" group for criteria $\mathbf{a}, \mathbf{b}$, and $\mathbf{c}$, when the data on phacoemulsification performed during the 5 -year follow-up were censored. The probabilities of success for criteria $\mathbf{a}, \mathbf{b}$, and $\mathbf{c}$ in the "trabeculectomy followed by phacoemulsification" and "trabeculectomy combined with phacoemulsification" groups were 66.7 and $43.0 \%$ for criterion A $(p=0.02), 64.6$ and $37.6 \%$ for criterion B ( $p<$ $0.01)$, and 54.2 and $22.6 \%$ for criterion $C(p<0.01)$, respectively. The data were compared using a log-rank test.

Trab = trabeculectomy; Phaco $=$ phacoemulsification 
Table 3 Hazard ratio analyzed using the multivariate Cox proportional hazards regression models

\begin{tabular}{|c|c|c|c|c|c|c|}
\hline \multirow[t]{3}{*}{ Factor } & \multicolumn{6}{|l|}{ Criteria } \\
\hline & \multicolumn{2}{|l|}{ A } & \multicolumn{2}{|l|}{ B } & \multicolumn{2}{|l|}{$\mathrm{C}$} \\
\hline & HR $(95 \% \mathrm{Cl})$ & $p$ Value & $\mathrm{HR}(95 \% \mathrm{Cl})$ & $p$ Value & HR (95\% Cl) & $p$ Value \\
\hline Age (years) & $0.97(0.95-0.99)$ & $0.02^{*}$ & $0.98(0.95-0.99)$ & $0.04^{*}$ & $0.98(0.96-1.00)$ & 0.07 \\
\hline Sex, F/M & $1,24(0.75-2.07)$ & 0.41 & $1.01(0.62-1.64)$ & 0.96 & $0.98(0.64-1.50)$ & 0.93 \\
\hline Preoperative IOP per mmHg & $0.94(0.91-0.98)$ & $<0.01^{*}$ & $0.96(0.93-0.99)$ & $0.04^{*}$ & $1.00(0.96-1.03)$ & 0.71 \\
\hline Conjunctival incision (Fornix/limbus based) & $0.93(0.55-1.55)$ & 0.77 & $0.96(0.58-1.58)$ & 0.86 & $1.06(0.69-1.64)$ & 0.80 \\
\hline EXG/POAG & $0.58(0.26-1.29)$ & 0.18 & $0.67(0.31-1.47)$ & 0.65 & $1.07(0.50-2.31)$ & 0.86 \\
\hline SG/POAG & $0.71(0.28-1.77)$ & 0.46 & $0.79(0.32-1.95)$ & 0.61 & $0.99(0.42-2.32)$ & 0.98 \\
\hline TLE combined with phaco & $2.31(1.22-4.38)$ & $0.01^{*}$ & $2.26(1.22-4.18)$ & $0.01^{*}$ & $2.23(1.30-3.82)$ & $<0.01^{*}$ \\
\hline Number of glaucoma medications & $1.04(0.80-1.35)$ & 0.77 & $1.06(0.82-1.35)$ & 0.68 & $1.12(0.90-1.40)$ & 0.31 \\
\hline
\end{tabular}

HR Hazard ratio, F Female, M Male, IOP Intraocular pressure, POAG Primary, EXG Exfoliation glaucoma, SG Secondary glaucoma due to uveitis, Posner PosnerSchlossman syndrome, TLE Trabeculectomy, Phaco phacoemulsification.

*Log-rank test

phacoemulsification or had lower cumulative probabilities of success than trabeculectomy alone. In contrast to trabeculectomy alone, combined phacoemulsification increases the risk of blood-aqueous barrier collapse. The flare value after phacoemulsification returns to normal only after 6 months, but the flare value after trabeculectomy returns to normal only after 4 weeks [17]. In

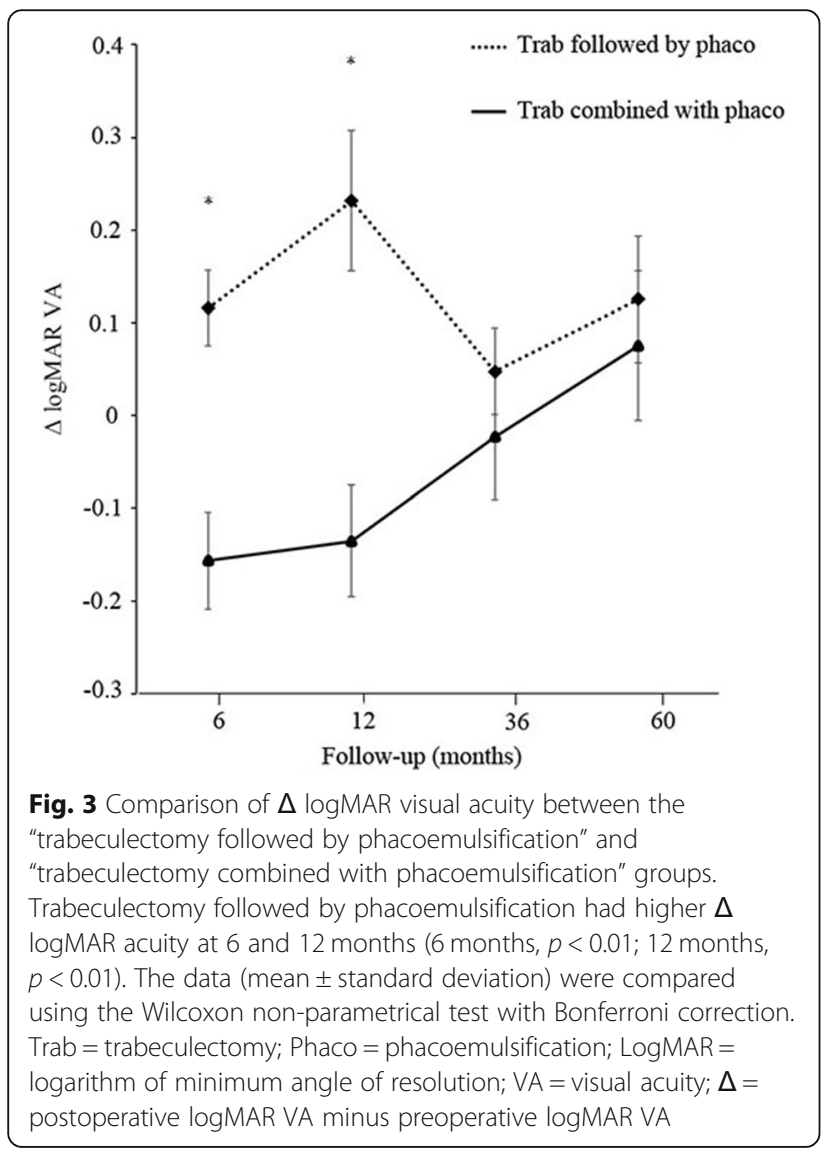

addition, various fibrogenic cytokines such as monocyte chemotactic protein-1 and interleukin- 8 are secreted after phacoemulsification [18, 19]. The prolonged inflammation eventually leads to bleb scarring [20].

Although significantly better outcomes in terms of $\Delta$ $\log$ MAR visual acuity were exhibited in the "trabeculectomy combined with phacoemulsification" group as compared with the "trabeculectomy followed by phacoemulsification" group at 6 and 12 months after surgery, the difference in $\Delta \log$ MAR visual acuity disappeared 12 months after surgery. In the "trabeculectomy combined phacoemulsification" group, visual acuity improved within a short-term follow-up period after surgery owing to the performance of cataract extraction. Similarly, Lockhead et al. [11] reported a mean gain in the number of lines in the Snellen visual acuity chart from baseline after trabeculectomy combined with phacoemulsification in patients with open-angle glaucoma with at least 1-year follow-up as compared with those who underwent trabeculectomy alone. Choy also retrospectively showed that patients who underwent phacotrabeculectomy had better visual acuity improvement for 3 months after surgery than those who underwent trabeculectomy alone [21]. Park et al. [6] also demonstrated that the mean visual acuity beyond the first postoperative month was significantly better than that at baseline after trabeculectomy combined with phacoemulsification. Similar to phacoemulsification alone, trabeculectomy combined with phacoemulsification also improved visual acuity [22, 23]. Moreover, trabeculectomy alone for phakic eyes triggered the occurrence of lens opacification after surgery $[2-4,24]$. The deterioration of visual acuity within 12 months after surgery in the "trabeculectomy followed by phacoemulsification" group seemed to be due to the progression of cataract. Thirty-three of the 48 eyes in the "trabeculectomy followed by phacoemulsification" group underwent phacoemulsification 
within 36 months. Therefore, the mean $\Delta \log$ MAR visual acuity was restored at 36 months after trabeculectomy. The difference in lens opacity between the two groups possibly led to the significant outcomes of $\Delta \log$ MAR visual acuity in the "trabeculectomy combined with phacoemulsification" group within 12 months. The $\Delta$ logMAR visual acuity at 60 months was almost equal between the two groups because of the gradual deterioration of visual acuity in the "trabeculectomy combined with phacoemulsification" group. As shown in Fig. 2, the surgical outcomes of the "trabeculectomy combined with phacoemulsification" group were significantly worse than those of the "trabeculectomy followed by phacoemulsification" group when data on phacoemulsification during the 5-year follow-up were censored. Compared with those in trabeculectomy alone, the uncontrolled postoperative IOPs in combined phacoemulsification might have resulted in the gradual deterioration of visual acuity over 5 years.

In the multivariate analysis using a Cox proportional hazards model, two additional risk factors of surgical failure were identified, namely younger age and lower preoperative IOP. Younger age has been considered a risk factor of trabeculectomy failure in many previous studies [25-28]. Vigorous healing response frequently occurs in individuals with a younger age, possibly owing to their thicker Tenon's capsule [29]. A $>20 \%$ IOP reduction in eyes with lower preoperative IOP is difficult to achieve without hypotony $[8,30]$.

This study had some limitations. First, as this was a cohort study, the participants were not randomly divided into two groups. In fact, the use frequency of fornixbased conjunctival flap was significantly higher in the "trabeculectomy combined with phacoemulsification" group. However, the Cox proportional hazards model showed that the surgical outcomes were not dependent on the base of the conjunctival flap. The incidence of secondary glaucoma due to uveitis was significantly higher in the "trabeculectomy combined with phacoemulsification" group. Eyes with uveitis were associated with cataract [31, 32]. The high incidence of cataract in the uveitic glaucomatous eyes might have caused the selection bias. Second, phacoemulsification, trabeculectomy, and trabeculectomy combined with phacoemulsification were not standardized. The technique used to perform these procedures depends on the discretion of each center. Hence, further prospective randomized studies should be conducted to resolve these limitations.

\section{Conclusion}

In conclusion, the present study showed no significant difference in cumulative probability of success between trabeculectomy followed by phacoemulsification and trabeculectomy combined with phacoemulsification for patients with primary open-angle glaucoma, exfoliation glaucoma, and glaucoma secondary to uveitis glaucoma. However, trabeculectomy combined with phacoemulsification appeared to have a lower cumulative probability of success than trabeculectomy alone. When the data on phacoemulsification during the 5-year follow-up were censored, younger age, lower preoperative IOP, and trabeculectomy combined with phacoemulsification were risk factors of surgical failure. The $\Delta \log M A R$ visual acuity in trabeculectomy followed by phacoemulsification was significantly higher in the early postoperative period than in trabeculectomy combined with phacoemulsification. However, the $\Delta \log$ MAR visual acuity had no significant differences between the two groups regardless of the timing of phacoemulsification.

\section{Abbreviations \\ CBIITS: Collaborative Bleb-related Infection Incidence and Treatment Study; IOP: Intraocular pressure; LogMAR: Logarithm of minimum angle of resolution}

\section{Acknowledgments}

The authors sincerely appreciate the Japan Glaucoma Society for providing them with the opportunity to perform this study using the CBIITS data.

\section{Authors' contributions}

SA: planned the study, analyzed data, and wrote the first and final drafts of the manuscript. KI, YO, and YT: collected and analyzed data, and contributed to the writing of the first draft of the manuscript. MI: planned the study, analyzed data, and contributed to the writing of the first and final drafts of the manuscript. The author(s) read and approved the final manuscript.

\section{Funding}

Not applicable.

Availability of data and materials

The datasets used and/or analyzed during the present study are available from the corresponding author on reasonable request.

\section{Declarations}

Ethics approval and consent to participate

The institutional review board of Fukui University Hospital, Fukui, Japan approved this study, and the certificate approval number is 20180085. Informed consent was obtained from all the patients participating in the CBIITS study.

\section{Consent for publication}

Not applicable.

\section{Competing interests}

None declared.

Received: 25 February 2021 Accepted: 7 April 2021

Published online: 24 April 2021

\section{References}

1. Ramulu PY, Corcoran KJ, Corcoran SL, Robin AL. Utilization of various glaucoma surgeries and procedures in Medicare beneficiaries from 1995 to 2004. Ophthalmology. 2007:114:2265-70.

2. AGIS investigators. The advanced glaucoma intervention study: 8 Risk of cataract formation after trabeculectomy. Arch Ophthalmol. 2001;119:1771-9.

3. Lazaro C, Benitez-del-Castillo JM, Castillo A, Garcia-Feijoo J, Macias JM, Garcia-Sanchez J. Lens fluorophotometry after trabeculectomy in open angle glaucoma. Ophthalmology. 2002;109:76-9. 
4. Adelman RA, Brauner SC, Afshari NA, Grosskreutz CL. Cataract formation after initial trabeculectomy in young patients. Ophthalmology. 2003;110: 625-9.

5. Rebolleda G, Muñoz-Negrete FJ. Phacoemulsification in eyes with functioning filtering blebs: a prospective study. Ophthalmology. 2002;109: 2248-55.

6. Park HJ, Kwon YH, Weitzman M, Caprioli J. Temporal corneal phacoemulsification in patients with filtered glaucoma. Arch Ophthalmol. 1997;115:1375-80

7. Husain R, Liang S, Foster PJ, Gazzard G, Bunce C, Chew PT, et al. Cataract surgery after trabeculectomy: the effect on trabeculectomy function. Arch Ophthalmol. 2012;130:165-70.

8. Arimura S, Iwasaki K, Gozawa M, Takamura Y, Inatani M. Trabeculectomy followed by phacoemulsification versus trabeculectomy alone: the Collaborative Bleb-Related Infection Incidence and Treatment Study. PLoS One. 2019;14:e0223439.

9. Ogata-Iwao M, Inatani M, Takihara Y, Inoue T, Iwao K, Tanihara H. A prospective comparison between trabeculectomy with mitomycin $C$ and phacotrabeculectomy with mitomycin C. Acta Ophthalmol. 2013;91:e500-1. https://doi.org/10.1111/aos.12133.

10. Lochhead J, Casson RJ, Salmon JF. Long term effect on intraocular pressure of phacotrabeculectomy compared to trabeculectomy. Br J Ophthalmol. 2003;87:850-2.

11. Flaxman SR, Bourne RRA, Resnikoff S, Ackland P, Braithwaite T, Cicinelli MV, et al. Global causes of blindness and distance vision impairment 1990-2020: a systematic review and meta-analysis. Lancet Glob Health. 2017;5:e1221-34. https://doi.org/10.1016/S2214-109X(17)30393-5

12. Yamamoto T, Sawada A, Mayama C, Araie M, Ohkubo S, Sugiyama K, et al. The 5-year incidence of bleb-related infection and its risk factors after filtering surgeries with adjunctive mitomycin C: collaborative bleb related infection incidence and treatment study 2. Ophthalmology. 2014;121:1001-6.

13. Yokota S, Takihara Y, Inatani M. Limbus- versus fornix-based trabeculectomy for open-angle glaucoma eyes with prior ocular surgery: the collaborative bleb-related infection incidence and treatment study. Sci Rep. 2015:5:9290.

14. Lakatos E. Sample sizes based on the log-rank statistic in complex clinical trials. Biometrics. 1988:44:229-41.

15. Sacchi M, Monsellato G, Villani E, Lizzio RAU, Cremonesi E, Luccarelli S, et al. Intraocular pressure control after combined phacotrabeculectomy versus trabeculectomy alone. Eur J Ophthalmol. 2021. https://doi.org/10.1177/112 0672121999997.

16. Donoso R, Rodriguez A. Combined versus sequential phacotrabeculectomy with intraoperative 5-fluorouracil. J Cataract Refract Surg. 2000;26:71-4.

17. Siriwardena D, Kotecha A, Minassian D, Dart JK, Khaw PT. Anterior chamber flare after trabeculectomy and after phacoemulsification. Br J Ophthalmol. 2000:84:1056-7.

18. Kawai M, Inoue T, Inatani M, Tsuboi N, Shobayashi K, Matsukawa A, et al. Elevated levels of monocyte chemoattractant protein-1 in the aqueous humor after phacoemulsification. Invest Ophthalmol Vis Sci. 2012;53:7951-60.

19. Inoue $\mathrm{T}$, Kawaji $\mathrm{T}$, Inatani M, Kameda T, Yoshimura N, Tanihara H. Simultaneous increases in multiple proinflammatory cytokines in the aqueous humor in pseudophakic glaucomatous eyes. J Cataract Refract Surg. 2012;38:1389-97.

20. Inoue T, Kawaji T, Tanihara H. Monocyte chemotactic protein-1 level in the aqueous humor as a prognostic factor for the outcome of trabeculectomy Clin Exp Ophthalmol. 2014:42:334-41.

21. Choy BNK. Comparison of surgical outcome of trabeculectomy and phacotrabeculectomy in Chinese glaucoma patients. Int J Ophthalmol. 2017; 10:1928-30.

22. Storr-Paulsen A, Pedersen $\mathrm{JH}$, Laugesen C. A prospective study of combined phacoemulsification-trabeculectomy versus conventional phacoemulsification in cataract patients with coexisting open angle glaucoma. Acta Ophthalmol Scand. 1998;76:696-9.

23. Vaideanu D, Mandal K, Hildreth A, Fraser SG, Phelan PS. Visual and refractive outcome of one-site phacotrabeculectomy compared with temporal approach phacoemulsification. Clin Ophthalmol. 2008;2:569-74.

24. Iwasaki K, Takamura Y, Arimura S, Tsuji T, Matsumura T, Gozawa M, et al. Prospective cohort study on refractive changes after trabeculectomy. J Ophthalmol. 2019. https://doi.org/10.1155/2019/4731653.

25. Molteno AC, Bosma NJ, Kittelson JM. Otago glaucomasurgery outcome study. Long-term results of trabeculectomy. 1976-1995. Ophthalmology. 1999;106:1742-50
26. Bevin TH, Molteno AC, Herbison P. Otago Glaucoma surgery outcome study: long-term results of 841 trabeculectomies. Clin Exp Ophthalmol. 2008;36: 731-7.

27. Ederer F, Gaasterland DA, Dally LG, Kim J, VanVeldhuisen PC, Blackwell B, et al. AGIS Investigators. The advanced glaucoma intervention study (AGIS): 13. Comparison of treatment outcomes within race: 10 -year results. Ophthalmology. 2004;111:651-64

28. Landers J, Martin K, Sarkies N, Bourne R, Watson P. A twenty-year follow-up study of trabeculectomy: risk factors and outcomes. Ophthalmology. 2012; 119:694-702.

29. Broadway DC, Chang LP. Trabeculectomy, risk factors for failure and the preoperative state of the conjunctiva. J Glaucoma. 2001;10:237-49.

30. Jampel HD, Solus JF, Tracey PA, Gilbert DL, Loyd TL, Jefferys JL, et al. Outcomes and bleb-related complications of trabeculectomy. Ophthalmology. 2012;119:712-22.

31. Okhravi N, Lightman SL, Towler HM. Assessment of visual outcome after cataract surgery in patients with uveitis. Ophthalmology. 1999;106:710-22.

32. Kump LI, Cervantes-Castañeda RA, Androudi SN, Foster CS. Analysis of pediatric uveitis cases at a tertiary referral center. Ophthalmology. 2005;112: 1287-92.

\section{Publisher's Note}

Springer Nature remains neutral with regard to jurisdictional claims in published maps and institutional affiliations.
Ready to submit your research? Choose BMC and benefit from:

- fast, convenient online submission

- thorough peer review by experienced researchers in your field

- rapid publication on acceptance

- support for research data, including large and complex data types

- gold Open Access which fosters wider collaboration and increased citations

- maximum visibility for your research: over $100 \mathrm{M}$ website views per year

At $\mathrm{BMC}$, research is always in progress.

Learn more biomedcentral.com/submissions 